\title{
Gastrite e ileíte a citomegalovírus como primeira manifestação de imunodeficiência comum variável num adolescente
}

\section{Cytomegalovirus gastritis and ileitis as presentation of common variable immunodeficiency in an adolescent}

Rev Port Imunoalergologia $2019 ; 27$ (3): $327-334$

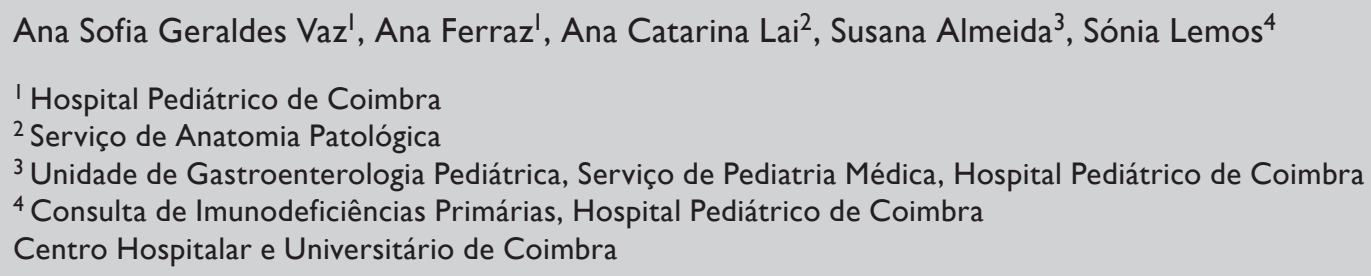

\section{RESUMO}

Objetivo: Descrever o caso de uma imunodeficiência comum variável (IDCV) cuja primeira manifestação foi diarreia crónica por infeção gastrointestinal por citomegalovírus (CMV). Esta é uma forma de apresentação rara e subdiagnosticada, cujo diagnóstico e intervenção precoces podem evitar a morbimortalidade associada a esta infeção. Descrição do caso: Adolescente de 16 anos com diarreia crónica desde os 10 anos e perda ponderal nos últimos 2 anos, sem antecedentes relevantes, referenciado à consulta com critérios diagnósticos de IDCV (diminuição de imunoglobulinas (Ig) G, A e M; ausência de anticorpos vacinais antiparotidite e antiHBs; exclusão de outras causas de hipogamaglobulinemia). Após início de terapêutica endovenosa com gamaglobulina, por manutenção de diarreia, realizou endoscopia digestiva alta e colonoscopia com biópsias, que mostrou gastrite e ileíte a CMV. Após terapêutica com ganciclovir endovenoso, verificou-se resolução clínica. Discussão: A infeção a CMV deve ser investigada em doentes com CVID e diarreia crónica simultaneamente com a exclusão de outras patologias gastrointestinais mais comuns, como infeção por Giardia lamblia, doença celíaca e doença inflamatória intestinal.

Palavras-chave: Citomegalovírus, diarreia, imunodeficiência comum variável. 


\section{ABSTRACT}

Objective: To describe the case of a common variable immunodeficiency (CVID) whose first manifestation was chronic diarrhea due to cytomegalovirus (CMV) gastrointestinal infection. This is a rare and underdiagnosed presentation, whose early diagnosis and intervention may prevent the morbidity and mortality associated with this infection. Case description: 16 year-old male adolescent with chronic diarrhea from the age of 10 years and body weight loss in the last 2 years, with no relevant past clinical history, referred to our consultation with diagnostic criteria for CVID (low levels of immunoglobulin (Ig) $G$, A and M; absence of anti-mumps and anti-hepatitis B surface antibodies; excluded other causes of hypogammaglobulinemia). After intravenous Ig replacement was initiated, diarrhea persisted, which led to perform high endoscopy and colonoscopy. Biopsy immunohistochemical analysis revealed CMV gastritis and ileitis. After intravenous ganciclovir therapeutic, clinical resolution was achieved. Discussion: CMV infection should be investigated in patients with CVID and chronic diarrhea simultaneously with the exclusion of more common gastrointestinal diseases such as Giardia lamblia infection, coeliac disease and inflammatory bowel disease.

Key-words: Cytomegalovirus, common variable immunodeficiency, diarrhoea.

\section{INTRODUÇÃO}

A imunodeficiência comum variável (IDCV) é uma imunodeficiência primária com uma prevalência estimada de I:100000 a I:10000, sendo o seu diagnóstico mais comum entre a segunda e quarta décadas de vida. A clínica é heterogénea, incluindo infeções, doenças autoimunes, linfoproliferativas e granulomatosas. As infeções bacterianas do trato respiratório são a forma mais comum de apresentação, nomeadamente em idade pediática ${ }^{\mathrm{l}-3}$.

A diarreia crónica ou síndrome de malabsorção como primeira manifestação de doença ocorre em 20 a $60 \%$ dos casos, devendo a doença celíaca e a doença inflamatória intestinal (DII) ser consideradas no seu diagnóstico diferencial. A causa infeciosa mais frequente da diarreia crónica nestes casos é a Giardia lamblia ${ }^{4,5}$.

A infeção sintomática por citomegalovírus (CMV) é praticamente limitada a indivíduos imunocomprometidos. A sua apresentação clínica mais frequente inclui febre, artralgias e mialgias, associada a citopenias transitórias, podendo manifestar-se também com envolvimento de órgão-alvo, com sintomas como diarreia e dor abdominal (infeção gastrointestinal) ou tosse e hipoxia (pneumonia). A doença gastrointestinal por CMV em doentes com IDCV é rara e o seu diagnóstico é frequentemente difícil e tardio. Alguns autores têm recentemente apontado para uma frequência superior desta infeção em doentes com IDCV, podendo esta ser uma entidade subdiagnosticada $^{6,7}$.

Apresenta-se o caso de um doente com IDCV que se apresentou com clínica exclusivamente gastrointestinal, confirmando-se posteriormente tratar-se de gastrite e ileíte por CMV.

\section{DESCRIÇÃO DO CASO}

Doente do sexo masculino referenciado à consulta de Imunodeficiências Primárias (IDP) aos 16 anos por diarreia crónica desde os 10 anos - dejeções líquidas, quatro a cinco vezes por dia, sem muco ou sangue ou 
dor abdominal associada. Aos 15 anos realizou colonoscopia em meio extra-hospitalar, cujo relatório descrevia ausência de alterações, tendo a diarreia sido atribuída a causa funcional. Aos 16 anos, aquando de internamento por apendicite aguda não complicada, pela persistência de diarreia, efetuou um estudo analítico que incluiu hemograma normal, coproculturas e exame parasitológico nas fezes (incluindo pesquisa de Giardia lamblia) negativos, anticorpo $\lg \mathrm{A}$ antitransglutaminase negativo, hormonas tiroideias normais, relação albumina/proteínas totais normal, ausência de proteinúria, eletroforese de proteínas plasmáticas com diminuição da fração gama, ausência de anticorpos vacinais da vacina da hepatite $B$ (VHB) e serologias do vírus da imunodeficiência humana negativas.

$\mathrm{Na}$ consulta de IDP confirmou-se que não havia antecedentes clínicos de relevo, à exceção de apendicite aguda. Os pais eram saudáveis e não consanguíneos e não havia história familiar sugestiva de IDP. Não tinha medicação habitual, não apresentava dismorfismos e o desen-

Quadro I. a. Doseamentos de imunoglobulinas em duas ocasiões com quatro semanas de intervalo; b. Imunofenotipagem de células B e T por citometria de fluxo

\begin{tabular}{|c|c|c|c|}
\hline I.a. Imunoglobulinas & $10^{\circ}$ & $2 .^{\circ}$ & Valores de referência \\
\hline $\lg G$ & 3,4 & 3,3 & $6,5-16,2 \mathrm{~g} / \mathrm{L}$ \\
\hline $\lg A$ & 0,25 & 0,25 & $0,57-5,43 \mathrm{~g} / \mathrm{L}$ \\
\hline $\lg M$ & 0,26 & 0,39 & $0,3-2,65 \mathrm{~g} / \mathrm{L}$ \\
\hline $\lg \mathrm{E}$ & 2,1 & - & $<\mid 8 \mathrm{UI} / \mathrm{L}$ \\
\hline I.b. Citometria de fluxo & \multicolumn{2}{|c|}{ Números absolutos (\%) } & Valores de referência* \\
\hline Linfócitos totais & \multicolumn{2}{|c|}{ 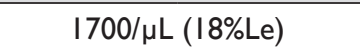 } & $1400-3300 / \mu \mathrm{L}$ \\
\hline $\begin{array}{l}\text { Células B } \\
\text { - naïve }(\lg M+\lg D+) \\
\text { - transicionais } \\
\text { - memória }(\mathrm{CD} 27+) \\
\text { Non-switched }(\lg M+) \\
\text { Switched }(\lg \mathrm{G}+/ \lg \mathrm{E}+/ \lg \mathrm{A}+)\end{array}$ & \multicolumn{2}{|c|}{$\begin{array}{c}286 / \mu \mathrm{L}(3 \% \mathrm{Li}) \\
-79 \%(\% \mathrm{~B}) \\
-1 \%(\% \mathrm{~B}) \\
-8 \%(\% \mathrm{~B}) \\
96 \%(\% \mathrm{BM}) \\
4 \%(\% \mathrm{BM}) \\
(\operatorname{lgG}+4 \%, \operatorname{lgA}+0 \%)\end{array}$} & 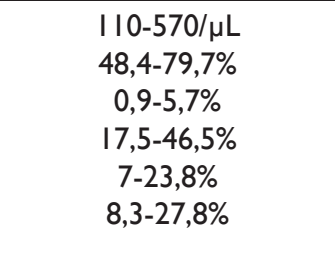 \\
\hline Células T & \multicolumn{2}{|c|}{ 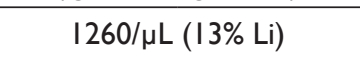 } & $1000-2200 / \mu \mathrm{L}$ \\
\hline $\begin{array}{l}\text { - CD4 } \\
\text { - naive } \\
\text { - memória } \\
\text { - efetoras/ativadas }\end{array}$ & \multicolumn{2}{|c|}{$\begin{array}{c}-857 / \mu \mathrm{L}(6 \mid \% \mathrm{~T}) \\
-43 \%(\% \mathrm{CD} 4) \\
-56 \%(\% \mathrm{CD} 4) \\
-1 \%(\% \mathrm{CD} 4)\end{array}$} & $\begin{array}{c}530-1300 / \mu L \\
33-66 \% \\
18-38 \% \\
4-11 \%\end{array}$ \\
\hline $\begin{array}{l}\text { - CD8 } \\
\text { - naive } \\
\text { - memória } \\
\text { - efetoras/ativadas }\end{array}$ & \multicolumn{2}{|c|}{$\begin{array}{l}-403 / \mu \mathrm{L}(35 \% \mathrm{~T}) \\
-65 \%(\% \mathrm{CD} 8) \\
-21 \%(\% \mathrm{CD} 8) \\
-10 \%(\% \mathrm{CD} 8)\end{array}$} & $\begin{array}{c}330-920 / \mu L \\
61-91 \% \\
4-23 \% \\
5-25 \%\end{array}$ \\
\hline Células NK & \multicolumn{2}{|c|}{134 (2\% Li) } & $70-480 / \mu \mathrm{L}$ \\
\hline
\end{tabular}

\%Le - percentagem do total de leucócitos; \%Li - percentagem do total de Infócitos; \%B - percentagem do total de células B; $\% \mathrm{~T}$ - percentagem do total de linfócitos T; \%CD4 - percentagem do total de linfócitos T CD4; \%CD8 - percentagem do total de linfócitos T CD8.

*Piatosa B, Wolska-Kusśnierz B, Pac M, Siewiera K, Gałkowska E, Bernatowska E. B cellsubsets in healthy children: Reference values for evaluation of B cell maturation process in peripheral blood. Cytometry B Clin Cytom 2010;78:372-8I. Shearer WT, Rosenblatt HM, Gelman RS, Oyomopito R, Plaeger S, Stiehm ER, et al. Lymphocyte subsets in healthy children from birth through 18 years of age: the Pediatric AIDS Clinical Trials Group PI009 study. J Allergy Clin Immunol 2003;1I2:973-80. 
volvimento psicomotor era normal. Foi constatada perda ponderal de $9 \%(5,5 \mathrm{~kg})$ nos últimos 3 anos, apresentando um índice de massa corporal (IMC) nos percentis 15-50. Os exames laboratoriais revelaram hemograma normal, diminuição de $\lg G$, $\lg A$ e $\lg M$ em dois doseamentos com intervalo de 4 semanas (Quadro la); ausência de anticorpos antiparotidite e antiHB; grupo sanguíneo A com título de isoemaglutininas anti-B baixo $(\mathrm{I} / \mathrm{I} 6)$, teste de transformação linfocitária com resposta proliferati- va normal aos mitogénios PHA e PWM e ao SPA, estudo de anticorpos anticitoplasma de neutrófilos e antinucleares negativo. Não foi possível avaliar a resposta a antigénios polissacarídicos (timoindependente). $\mathrm{Na}$ citometria de fluxo (Quadro lb), o estudo fenotípico das células B do sangue periférico revelou diminuição das células de memória $\mathrm{CD} 27+$ ( $8 \%$ dos linfócitos $\mathrm{B})$ e uma baixa acentuada de linfócitos $B$ de memória switched (4\%). A fenotipagem das células $T$ mostrou um aumento das células
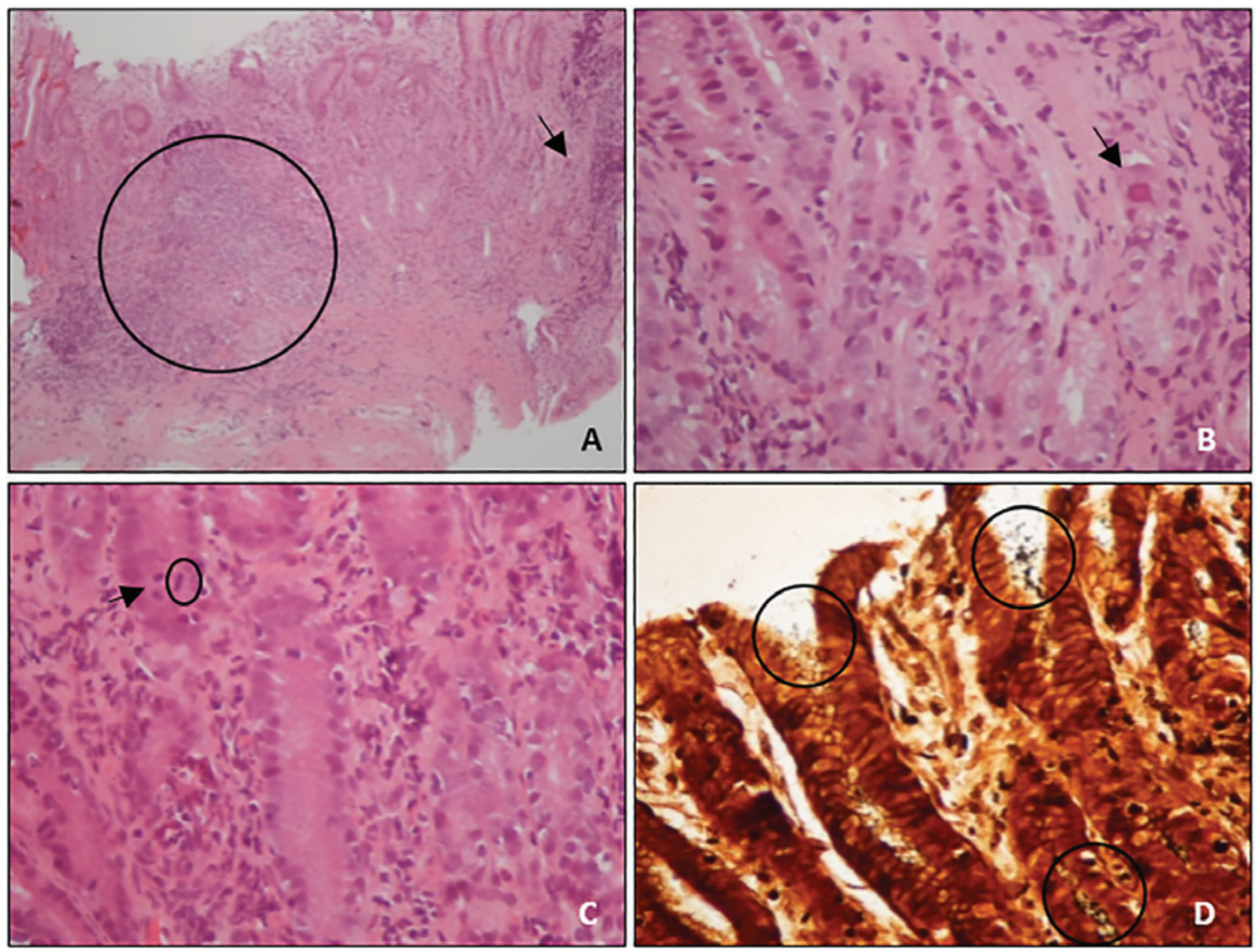

Figura I. Antro gástrico. (A) Gastrite crónica atrófica ligeira com infiltrado inflamatório misto moderado, com esboço de agregados linfoides (círculo) e célula volumosa, sugerindo inclusão por citomegalovirus (seta); hematoxilina e eosina (HE), I00x. (B) Célula com citoplasma granular e volumoso e núcleo anfófilo - inclusão citomegálica (seta); HE, 400x. (C) Polimorfonucleares neutrófilos a permear o epitélio das criptas (seta), traduzindo atividade e algumas mitoses (círculo); HE, 400x. (D) Colonização ligeira por microrganismos de tipo Helicobacter pylori, aderentes ao epitélio, na superfície e na profundidade, das fóveas (círculos). Whartin-Starry, 400x. 
CD4 de memória (56\%) e uma diminuição das células CD4 efetoras (I\%).

Foi colocada a hipótese de IDCV e iniciou terapêutica endovenosa com gamaglobulina (GGEV), com periodicidade mensal (dose de $600 \mathrm{mg} / \mathrm{kg}$ ). Verificou-se melho- ria do padrão da diarreia (diminuição da frequência das dejeções) apenas nos três dias seguintes à administração de GGEV, com posterior reagravamento. Foi colocada a hipótese de doença celíaca associada a IDCV. Dada a deficiência de $\lg A$ e a terapêutica mensal com gamaglo-
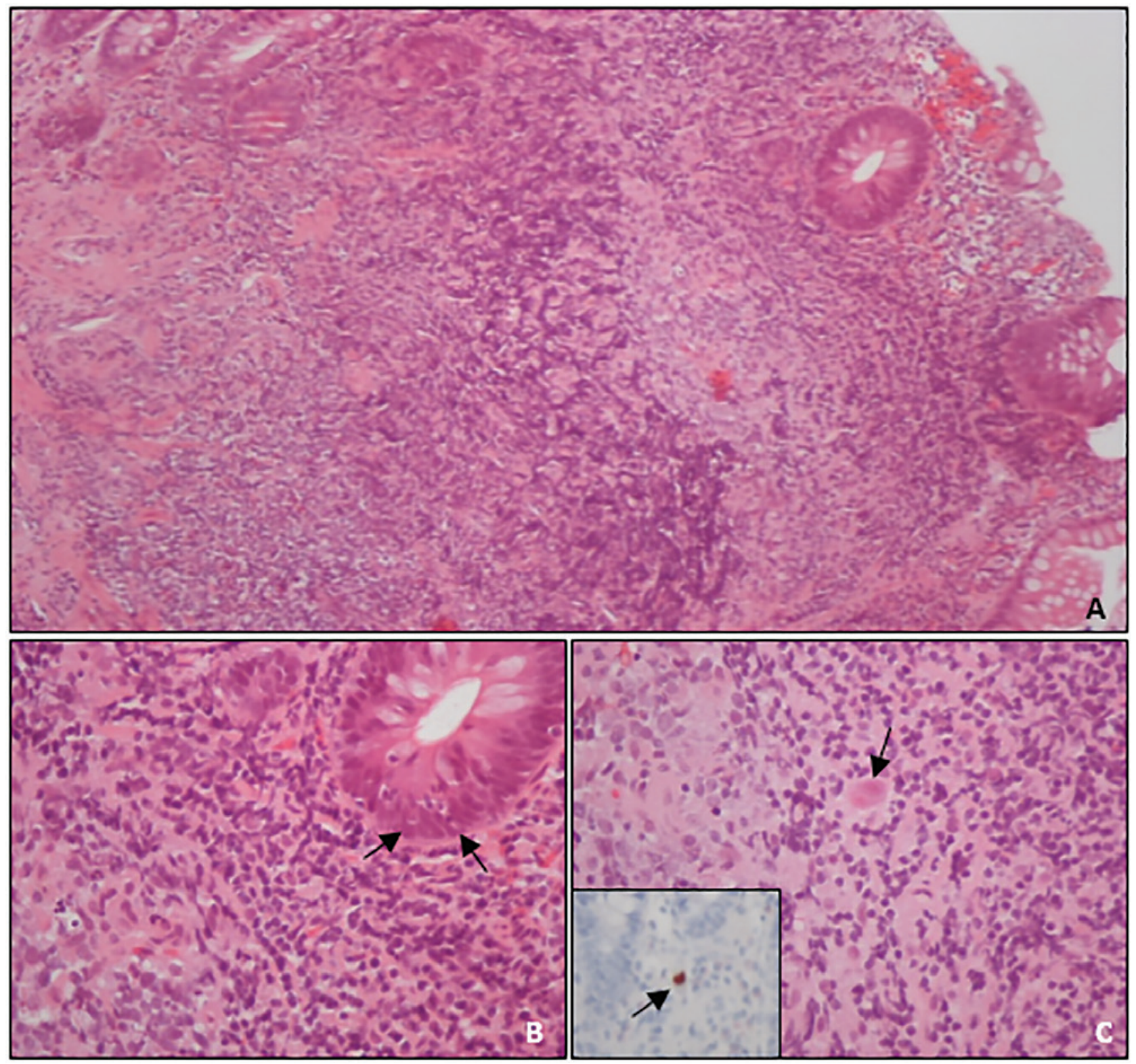

Figura 2. Íleon terminal. (A) Alteração da arquitetura das vilosidades e criptas, na lâmina própria, com infiltrado inflamatório misto e tecido linfoide associado à mucosa. HE, 100x . (B) Infiltrado inflamatório linfoplasmocitário e com polimorfonucleares neutrófilos intraepiteliais (seta). HE, 400x. (C) Célula com citoplasma amplo e núcleo anfófilo e volumoso, sugestiva de inclusão de CMV (seta). (HE, 400x). No canto inferior esquerdo, confirmação com o anticorpo específico para CMV (seta). 
bulina, com consequente baixa fiabilidade do rastreio serológico, foi realizada endoscopia digestiva alta (EDA) e colonoscopia. As biópsias seriadas do tubo digestivo revelaram gastrite crónica antral com atrofia, colonização moderada por Helicobacter pylori (Hp) e inclusões citomegálicas (Figura I), duodenite com atrofia vilositária, ileíte terminal com infiltrado linfoplasmocitário e inclusões citomegálicas (Figura 2) e pancolite crónica difusa com infiltrado inflamatório misto.

Foram pesquisados os haplotipos HLA de risco para doença celíaca (DQ2/DQ8), com resultados negativos.

Iniciou tratamento para infeção a CMV com ganciclovir endovenoso na dose de $10 \mathrm{mg} / \mathrm{kg} / \mathrm{dia}$, cada $12 \mathrm{~h}$, que cumpriu durante 14 dias, tendo continuado a terapêutica antiviral com valganciclovir oral na dose de $450 \mathrm{mg}$, cada I $2 \mathrm{~h}$, no domicílio, durante 4 semanas. Simultaneamente, iniciou terapêutica de erradicação do Hp com amoxicilina, metronidazol e omeprazol (antibioterapia 14 dias, omeprazol quatro semanas). Foi administrada cianocobalamina intramuscular por doseamentos de vitamina BI2 baixo (183pg/mL; normal:200-950) e ácido fólico no limite inferior (3,2 ng/mL; normal:3-17). Durante o internamento foi notada melhoria do padrão das dejeções (menor número/dia, maior consistência das fezes, ausência de dejeções noturnas), melhoria do apetite e aumento ponderal de $3 \%(I, 3 \mathrm{~kg})$ em duas semanas.

Após a alta, manteve padrão de uma dejeção diária com fezes moldadas e verificou-se aumento ponderal (IMC P50-85) nos meses seguintes.

Realizou EDA de controlo com biópsia gástrica, sem evidência de infeção a CMV, mantendo infeção a Hp. Iniciou segunda tentativa de erradicação do Hp com esomeprazol, ampicilina, clindamicina e metronidazol, com erradicação confirmada por teste respiratório com pesquisa de Hp. A colonoscopia de controlo mostrou biópsia ileal também sem evidência de infeção a CMV e com hiperplasia de tecido linfoide associado à mucosa e cólon com infiltrado inflamatório mononuclear ligeiro.

Manteve seguimento em consulta de IDP e Gastroenterologia, tendo repetido doseamento de $\lg G, \lg A$ e $\lg M$ após resolução da diarreia, verificando-se manutenção de níveis baixos, excluindo hipogamaglobulinemia por perda intestinal, mantendo necessidade de terapêutica com GGEV, com periodicidade mensal, na dose de $600 \mathrm{mg} / \mathrm{kg}$.

Nos três anos seguintes registaram-se cerca de duas infeções respiratórias por ano, com boa evolução sob amoxicilina+ácido clavulânico oral e sem necessidade de internamento.

\section{DISCUSSÃO}

A IDCV é a imunodeficiência primária sintomática mais comum e é caracterizada por níveis baixos de lgG, IgA e/ou IgM, com reduzida produção de anticorpos e falha na resposta a antigénios polissacarídicos e proteicos. As manifestações mais frequentes em idade pediátrica incluem infeções respiratórias bacterianas graves e/ou recorrentes, incluindo pneumonia, sinusite e otite média aguda, assim como diarreia crónica e má progressão ponderal. A diarreia crónica é mais frequentemente secundária a infeção por Giardia lamblia, hiperplasia linfoide nodular e atrofia vilositária, originando quadro de malabsorção. A doença inflamatória intestinal (DII) e a celíaca são outras causas de malabsorção e diarreia associadas a IDCV 4,5 .

Devido à sua heterogeneidade, o diagnóstico de IDCV é desafiante e de exclusão. Vários critérios de diagnóstico têm sido propostos. Segundo o Consenso Internacional ICON ${ }^{8}$, este doente preenche os critérios de IDCV: idade superior a quatro anos, hipogamaglobulinemia $\lg G$ associada a baixos níveis de $\lg A$ e $\lg M$ em duas ocasiões, excluídas outras causas de hipogamaglobulinemia, como iatrogenia medicamentosa, infeção por $\mathrm{VIH}$ e enteropatia perdedora de proteínas, e ausência de resposta a um antigénio timodependente ou a um antigénio timoindependente. Neste caso, o doente não apresentava resposta a dois antigénios vacinais timodependentes (VHB e parotidite) e tinha baixo título de isoemaglutininas. 
caso que apresentamos tem a particularidade de se ter manifestado por clínica exclusivamente gastrointestinal (gastrite a Hp, gastrite e ileíte a CMV), sem história de infeções respiratórias. São raras as descrições de casos de IDCV com manifestações gastrointestinais isoladas e/ou víricas. Por outro lado, infeção a CMV é também mais frequente quando existem alterações da imunidade celular, o que se verificou neste doente. No entanto, mais recentemente, alguns estudos identificaram com maior frequência a infeção a CMV nestes doentes, apontando para um provável subdiagnóstico ${ }^{6,7}$. Outro aspeto que tem vindo a ser associado à infeção por CMV na IDCV, e que se verifica no caso apresentado, refere-se à tendência para uma colonização pandigestiva do tubo gastrointestinal, ao contrário da sua habitual limitação à mucosa do cólon na infeção por CMV na DII7.

A prevalência de infeção por $\mathrm{Hp}$ em doentes com IDCV é semelhante à verificada na população geral. No entanto, a infeção por Hp nestes doentes está frequentemente associada a gastrite atrófica e pode induzir displasia gástrica e maior risco de cancro gástrico, sendo relevante o seguimento destes doentes no que se refere à realização de endoscopias segundo protocolos e à avaliação dos níveis de vitamina $\mathrm{BI}$, que, no caso descrito, estavam baixos, com necessidade de suplementação por via parentérica?.

No caso descrito, as coproculturas e exame parasitológico nas fezes (incluindo pesquisa de Giardia lamblia) negativos excluíram estas causas infeciosas. Outras causas infeciosas, como norovírus, adenovírus e outros enterovírus poderiam também ser excluídas. A refratariedade da diarreia à terapêutica com GGEV suscitou outras hipóteses diagnósticas, como a doença celíaca (neste caso não excluída com fiabilidade pela negatividade do anticorpo $\lg \mathrm{A}$ antitransglutaminase, devido aos baixos níveis de IgA) e a DII, levando à realização de endoscopia digestiva alta e colonoscopia. A identificação do $\mathrm{CMV}$ por análise imunoistoquímica nas biópsias de tecido do tubo digestivo permitiu o diagnóstico da infeção e seu tratamento de forma relativamente precoce, comparativamente a outros publicados, evitando a progressão da doença e o surgimento de complicações associadas e morbilidade e mortalidade elevadas, como desnutrição grave com necessidade de alimentação parentérica ou a formação de lesões ulcerativas, estenoses intestinais e fístulas pararetais ${ }^{6,10}$. No entanto, o diagnóstico poderia ter sido ainda mais precoce se na primeira colonoscopia realizada, aos 15 anos, tivessem sido efetuadas biópsias e respetivo estudo histológico. Assim, será de interesse realizar o despiste de infeção por CMV simultaneamente com o despiste de DII, sendo para tal fundamental a realização de biópsias do tubo digestivo aquando do estudo endoscópico, mesmo nos casos em que a mucosas não apresentem aparentes alterações macroscópica.

Em conclusão, gostaríamos de reforçar a necessidade de considerar e investigar a possibilidade de infeção a CMV em casos de diarreia crónica em doentes com IDCV, de forma a possibilitar um diagnóstico precoce, com importantes implicações em termos de prognóstico.

\section{Conflito de interesses}

Os autores declaram que não existem conflitos de interesse.

Contacto:

Ana Sofia Geraldes Vaz

Hospital Pediátrico de Coimbra, Consulta de Imunodeficiências, Avenida Afonso Romão 3000-602, Coimbra, Portugal anasgvaz@hotmail.com

\section{REFERÊNCIAS}

I. Cunningham-Rundles C, Bodian C. Common variable immunodeficiency: clinical and immunological features of 248 patients. Clin Immunol 1999;92:34-48.

2. Yong PF, Thaventhiran JE, Grimbacher B. "A rose is a rose is a rose," but CVID is Not CVID common variable immune deficiency (CVID), what do we know in 20II? Adv Immunol 201 I; III:47-107.

3. Grimbacher B; ESID Registry Working Party. The European Society for Immunodeficiencies (ESID) registry 2014. Clin Exp Immunol 2014;178 Suppl I:18-20. 
4. Patuzzo G, Barbieri A, Tinazzi E, Veneri D, Argentino G, Moretta $F$, et al. Autoimmunity and infection in common variable immunodeficiency (CVID). Autoimmun Rev 2016; 15:877-82.

5. Jørgensen SF, Reims HM, Frydenlund D, Holm K, Paulsen V, Michelsen $\mathrm{AE}$, et al. A cross-sectional study of the prevalence of gastrointestinal symptoms and pathology in patients with common variable immunodeficiency. Am J Gastroenterol 2016; III: 1467-75.

6. Kralickova P, Mala E, Vokurkova D, Krcmova I, Pliskova L, Stepanova $\mathrm{V}$, et al. Cytomegalovirus disease in patients with common variable immunodeficiency: three case reports. Int Arch Allergy Immunol 2014;163:69-74.

7. Costantino G, Mondello P, Previti M, Fries W, Villanacci V. Pan-digestive tract colonization by cytomegalovirus in common variable immunodeficiency. Int Arch Allergy Immunol 20I4;164: $30-$ I.

8. Bonilla FA, Barlan I, Chapel H, Costa-Carvalho BT, Cunningham-Rundles C, de la Morena MT, et al. International Consensus Document (ICON): common variable immunodeficiency disorders. J Allergy Clin Immunol Pract 2016;4:38-59.

9. Uzzan M, Ko HM, Mehandru S, Cunningham-Rundles C. Gastrointestinal disorders associated with common variable immune deficiency (CVID) and chronic granulomatous disease (CGD). Curr Gastroenterol Rep 2016;18:17.

10. Tahan V, Dobrucali A, Canbakan B, et al. Cytomegalovirus infection of gastrointestinal tract with multiple ulcers and strictures, causing obstruction in a patient with common variable immunodeficiency syndrome. Dig Dis Sci 2000; 45: 178I-5. 